\title{
ERRATA
}

\section{Electron Theory of Long-Wavelength Concentration Fluctuations in Liquid Metal Alloys.}

WANG LI

Intermational School for Advanced Studies - Trieste, Italia

M. P. Tosi

Dipartimento di Fisica Teorica dell'Università - Trieste, Italia

International Centre for Theoretical Physics - Trieste, Italia

(Nuovo Cimento D, 11, 1509 (1989))

PACS 61.20.Gy - Statistical theories of liquid structure.

PACS 99.10 - Errata.

The value of the interchange free energy $w$ in Na-K alloys, which is obtained by fitting the experimental data on $S_{\mathrm{cc}^{\prime}}$, is $w \approx 1.1 k_{\mathrm{B}} T$ instead of $w \approx 11 k_{\mathrm{B}} T$ as was misprinted in the article. Hence, the claim made in the article that the elastic contribution to $w$, which is of order $10 k_{\mathrm{B}} T$, must be largely cancelled by the electronic charge transfer contribution. 